Revue européenne des migrations internationales

vol. $21-n^{\circ} 1 \mid 2005$

Femmes, genre, migration et mobilités

\title{
La place des femmes dans les réseaux migrants roumains
}

Swanie Potot

\section{(2) OpenEdition}

1 Journals

Édition électronique

URL : https://journals.openedition.org/remi/2335

DOI : $10.4000 /$ remi.2335

ISSN : $1777-5418$

Éditeur

Université de Poitiers

Édition imprimée

Date de publication : 29 mai 2005

Pagination : 243-257

ISBN : 2-911627-39-3

ISSN : 0765-0752

Référence électronique

Swanie Potot, «La place des femmes dans les réseaux migrants roumains », Revue européenne des migrations internationales [En ligne], vol. $21-\mathrm{n}^{\circ} 1$ | 2005, mis en ligne le 08 septembre 2008, consulté le 14 avril 2022. URL : http://journals.openedition.org/remi/2335; DOI : https://doi.org/10.4000/remi. 2335

Ce document a été généré automatiquement le 14 avril 2022.

() Université de Poitiers 


\title{
La place des femmes dans les réseaux migrants roumains
}

\author{
Swanie Potot
}

1 Les circulations migratoires transeuropéennes observées ces dernières années (Diminescu, 2003 ; Morokvasic et Rudolph, 1996 ; Wallace et Stola, 2001) reposent sur des schémas migratoires dans lesquels hommes et femmes mettent en œuvre des stratégies similaires et participent à des activités semblables à l'étranger. Ces pratiques invitent à interroger plus avant le rôle différencié que peuvent jouer les femmes et les hommes au sein de dispositifs migratoires communs (Morokvasic, 2003).

2 La réflexion présentée ici s'appuie sur une série d'enquêtes menées auprès de migrantes et migrants temporaires roumains sur différents pôles migratoires en Europe occidentale et dans des zones de départ et de retour en Roumanie. Ces terrains d'étude ont été conduits dans le cadre d'une recherche sur la circulation migratoire pratiquée de façon croissante par de nombreux Roumains au cours des années quatrevingt-dix pour résister à la crise économique qui frappe leur pays depuis la fin de la dictature de N. Ceausescu (Potot, 2003). Il s'agit de migrations temporaires durant lesquelles les migrants alternent des périodes de travail peu qualifié à l'étranger et des périodes - de quelques mois à une année voire plus - en Roumanie (Lagrave et Diminescu, 1999; Sandu, 2000). Ces séjours à l'étranger visent à améliorer les conditions de vie des migrants sans se couper durablement de leur existence sociale chez eux ${ }^{1}$.

3 Les réseaux sociaux qui servent de support aux migrations transnationales de travail sont numériquement dominés par les hommes, mais, au cours de ces dernières années, un nombre toujours plus important de femmes ont participé à ces migrations temporaires. Or, tout en faisant usage des mêmes stratégies migratoires et des mêmes réseaux sociaux, il apparaît que ces dernières ne vivent pas cette expérience de la même façon que leurs homologues masculins. C'est en deuxième analyse, suite à une prise de conscience progressive de la pertinence sociologique d'une approche en termes de genre, que j'ai repris les données de première main recueillies au cours de plusieurs années d'enquête. Une telle analyse soulignera notamment que le genre 
n'influence pas seulement les pratiques liées à la migration, mais intervient également au niveau social et symbolique.

\section{Târgoviste, Nice, Londres : évolution d'un parcours migratoire}

4 Afin de saisir le sens des données recueillies, il est nécessaire, dans un premier temps, de revenir sur le travail de terrain effectué pour présenter brièvement le schéma général des mouvements migratoires. Les différentes enquêtes que j'ai menées portent sur deux réseaux migratoires : l'un s'est déployé, à partir de 1995, entre Târgoviste², ville moyenne de la région de Bucarest, et Nice, avant de se déplacer, après 1998-1999, vers Londres. L'autre s'est constitué durant la même période, entre le département rural de Téléorman, dans la plaine du Danube, et la province d'Alméria, dans le sud de l'Espagne (Voir schéma).

Schéma des circulations étudiées

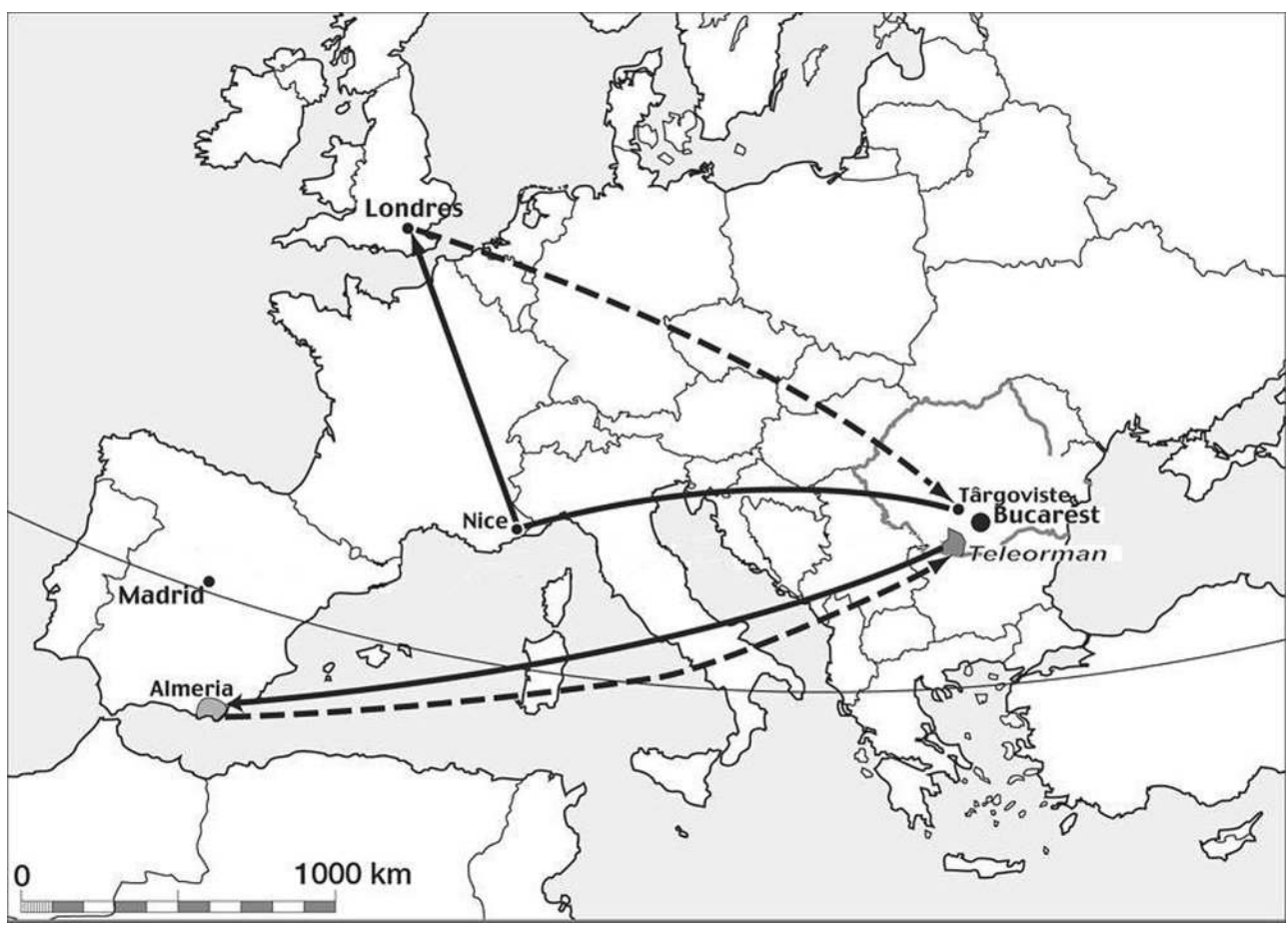

5 Les migrations observées reposent sur des dispositifs économiques instables et ne cessent de se transformer et de se réinventer (Péraldi, 2001a). Au cours de ces quatre années, la migration de Târgoviste a connu son âge d'or à Nice, puis son déclin a annoncé une phase de tâtonnements durant lesquels les migrants ont cherché d'autres pôles migratoires ailleurs en Europe, avant de se réorienter massivement vers la capitale britannique. Ce sont ces différentes étapes que j'ai pu suivre au cours de plusieurs enquêtes menées à Nice, Târgoviste et Londres.

6 Les premières observations menées sur la Côte d'Azur ont permis de conclure qu'il se trouvait, dans la région niçoise, au moins une centaine de jeunes roumains et roumaines, venus pour travailler durant quelques mois, voire une année, avant de repartir vers leur ville d'origine. La plupart étaient originaires de la même ville de 
Roumanie et avaient manifestement des liens entre eux. C'est grâce, non pas à une forte solidarité, mais à des collaborations au coup par coup que les migrants parvenaient à se déplacer en Europe et à y travailler tandis que la législation restreignait ces possibilités ${ }^{3}$ (Tarrius, 1992 ; Péraldi, 2001b). En répliquant et en perfectionnant au cours du temps l'expérience de quelques pionniers, ils avaient réussi à construire, dans la région niçoise, une "niche ethnique" (Waldinger, 1994) spécifique à la migration roumaine. Ce réseau se concentrait presque exclusivement sur cette zone géographique et notamment autour de l'activité de vente d'un journal de rue distribué à Nice ${ }^{4}$. Étant donné ma position - une femme enquêtant dans un environnement majoritairement masculin - c'est surtout avec des migrantes que j'entretenais des relations suivies.

7 À partir de 1995 et jusqu'au déclin de cette migration à la fin de la décennie, j'estime qu'environ trois cents migrants de Târgoviste se sont rendus sur la Côte d'Azur au moins une fois. Durant la période d'enquête, j'ai rencontré ou obtenu des informations détaillées sur environ cent vingt personnes originaires de cette ville. J'ai également enquêté à Târgoviste, ville de départ des migrants et migrantes ${ }^{5}$. Le milieu auquel je m'intéressais, dont les limites restaient très floues, comprenait une centaine de personnes, qui avaient déjà effectué des séjours à l'étranger ou qui, se trouvant sur le point de partir, fréquentaient régulièrement les migrants. Ce groupe comprenait environ $10 \%$ de femmes avec lesquelles j'avais des contacts réguliers ${ }^{6}$.

8 À la fin de la décennie, ce réseau s'est progressivement déplacé vers Londres - où la répression à l'égard des clandestins était jugée moins sévère - qui est depuis devenue sa destination principale ${ }^{7}$. Si l'on compare avec la situation étudiée en France, il apparaît que les conditions juridiques, sociales et économiques ont une influence importante sur le comportement collectif des migrants. Le nombre important de femmes et de couples mariés est notamment un indicateur de ces changements de statut du migrant. En France, la possibilité de se voir expulser à tout moment et la difficulté à trouver un emploi stable (même illégal) sélectionnaient les candidats en fonction de leurs moyens, leur motivation et leur débrouillardise. Face aux difficultés rencontrées, il arrivait en effet que des migrants se trouvent contraints de rentrer en Roumanie sans être parvenus à tirer profit de la migration; l'échec pouvait ainsi s'avérer très coûteux. Avec le déplacement vers la Grande-Bretagne, la situation étant moins "risquée ", des candidats au départ moins audacieux ou bien ceux possédant moins de ressources se sont alors joints au mouvement. Un certain nombre de femmes profitèrent également de cette facilitation pour entrer à leur tour dans le processus migratoire.

\section{De Téléorman à Alméria : un mouvement continuel}

9 L'autre série d'enquêtes concerne une migration indépendante de la première, qui s'est développée entre un département du Sud roumain et la province agricole d'Alméria, en Espagne. Dans cette région, un nombre important de Roumains et Roumaines, plusieurs milliers selon mes informateurs, trouvent à se faire embaucher comme ouvriers temporaires dans les serres de fruits et légumes. Comme dans le cas précédent, la grande majorité de ces travailleurs est originaire d'une même région de Roumanie, le département de Téléorman, et la plupart d'entre eux trouvent à se loger ou à se faire employer illégalement en Espagne grâce au soutien ou aux informations de compatriotes qui les ont précédés dans la migration'. 
10 Les enquêtes ont dévoilé que la présence des migrants et de nombreuses migrantes (d'après mes estimations, on compterait environ $25 \%$ à $30 \%$ de femmes), dans la région, reposait sur le même type de pratiques et de stratégies migratoires que celles, rencontrées précédemment. Cependant, la situation du travailleur étranger se trouvait quelque peu différente dans la mesure où, derrière le discours sécuritaire des autorités espagnoles, se cache une politique complaisante à l'égard des clandestins qui sont utiles - voire indispensables - à l'expansion de l'économie locale (Checa, 1999). De plus, dans le sud de l'Espagne, les Roumains sont des migrants très appréciés face à leurs homologues marocains qui, eux, souffrent de racisme (Potot, 2003). En outre ces conditions particulières jouent fortement en faveur de la féminisation de cette migration. Un terrain d'étude a été conduit dans la région d'origine de ces migrants, afin de comprendre comment cette circulation migratoire était vécue dans le département rural de Téléorman'. Les données recueillies ont notamment fait apparaître que, contrairement aux jeunes originaires de Târgoviste les personnes qui partent n'attendent pas de revenir pour commencer à dépenser les gains de la migration. Bien souvent, ces derniers sont utilisés au fur et à mesure, dans la construction d'une maison pour leur famille ou investis pour payer les études des enfants. Les migrants qui partent seuls étant plus souvent des hommes, ce sont alors les femmes de ces derniers qui gèrent sur place les dépenses qu'autorise la migration. Les départs tout comme les retours des migrants sont dans ce cas longuement discutés en couple, en fonction des impératifs financiers du ménage et de la bonne ou de la mauvaise gestion de ces rentes dans le village d'origine. Cette situation souligne que même lorsqu'elles ne partent pas, les femmes des migrants jouent un rôle non négligeable dans le processus migratoire.

\section{Le réseau migrant au féminin}

11 La présence des femmes dans ces réseaux migrants ne se cantonne pas à une simple représentation par procuration. C'est tout d'abord en Roumanie, lorsque l'on envisage de partir pour la première fois, que le voyage se prépare et que le parcours des femmes se singularise.

\section{Partir avec le soutien d'un homme}

12 En dehors d'accords ponctuels négociés entre États, les migrations de travail dans l'Union Européenne ne sont pas accessibles, légalement, aux ressortissants roumains. C'est pourquoi les soutiens de la part d'autres migrants sont tout à fait indispensables. Pour un homme, il est cependant possible d'organiser un premier départ sans avoir de proches à l'étranger ou qui en soient revenus. Il lui faudra pour cela entrer en contact, en Roumanie, avec des personnes impliquées dans le milieu de la migration, qui pourront l'informer ou lui vendre des moyens de se rendre en Occident.

13 Il existe en effet, dans ce pays, un véritable marché de l'émigration, qui concerne des procédures aussi bien légales qu'illégales, mais dont le cadre est toujours informel. On peut ainsi acheter un visa de travail, obtenir de faux papiers ou bien apprendre comment se rendre au Royaume-Uni sans être arrêté à la frontière. Cependant, l'informalité et le nombre important de candidats rendent ces recours très incertains : les escroqueries sont fréquentes et nombreux sont les migrants qui racontent en avoir 
été victimes au moins une fois avant de parvenir à partir. De ce fait, un candidat au départ augmente ses chances de succès lorsqu'il peut mobiliser un réseau de connaissances lié à la migration. Il en va de même lors du séjour à l'étranger : plus le nouveau venu sera épaulé par des pairs plus sa migration sera facilitée. Toutefois, même un migrant isolé, au début, sera amené à rencontrer des pairs au cours de son séjour à l'étranger. Chaque région de départ en Roumanie privilégie quelques pôles migratoires; en conséquence, il est toujours possible, avant de partir, d'obtenir quelques renseignements sur ces destinations. Par exemple, la plupart des habitants du département de Téléorman savent que s'ils se rendent en Espagne, ils pourront trouver à se faire embaucher dans l'agriculture dans la province d'Alméria. Ce type d'informations très générales conduit alors les novices qui ne bénéficient pas d'aide à se rapprocher des autres migrants de leur région en situation migratoire. Les contacts répétés, dans les situations de travail, mais aussi parce qu'ils logent dans les mêmes quartiers ou parce qu'ils fréquentent les mêmes services sociaux, sont alors à la base de relations qui se tissent dans l'espace de la migration. Ces liens seront ensuite mobilisés en cas de besoin. Ainsi, il peut arriver qu'un homme envisage seul un départ puis entre, à l'étranger, au sein d'un réseau relationnel propre à la migration, assurant de plus en plus ses démarches grâce au soutien de pairs.

En revanche, cette façon de procéder n'est pas celle des femmes que l'on rencontre dans ces réseaux. En effet, celles-ci n'entrent quasiment jamais seules dans le processus migratoire; la décision de partir fait, en général, l'objet de discussions et de négociations avec des proches (Pheterson, 1994). Les femmes qui partent travailler à l'étranger sont rarement affranchies de toute famille (Magyari-Vincze, 2004) et, bien souvent, elles sont mariées ou ont un petit ami qui interviendra de façon importante dans la préparation d'un premier départ ${ }^{10}$.

Il arrive que le conjoint soit parti en premier et que la femme parte le rejoindre à l'étranger. La décision intervient alors au sein du couple. Elle peut être motivée par deux facteurs : le mari peut considérer que sa situation est suffisamment stable là où il se trouve et propose à sa femme de le retrouver afin d'éviter une séparation trop longue. Ce type de rapprochement est alors proche du regroupement familial, même s'il n'implique pas une installation de longue durée. Mais cette situation s'explique aussi souvent par un autre calcul : être deux à l'étranger permet d'écourter le voyage. En effet, dans le cas de couples, la migration sert souvent à poursuivre un objectif précis, même s'il peut se résumer à amasser un certain pécule pour vivre plus aisément le quotidien en Roumanie. Alors, le départ de la femme vise à réaliser plus rapidement cet objectif afin de rentrer au pays. Dans le discours des migrants, ces deux raisons sont souvent concomitantes. Dans tous les cas, le regroupement, qui peut être prévu dès le début ou intervenir bien après le départ du mari, n’a lieu que lorsque celui-ci est relativement à l'aise dans la situation migratoire. Cela ne signifie pas seulement qu'il ait un emploi et un logement mais également qu'il ait acquis une place au sein du réseau migrant et qu'il sache «se débrouiller » à l'étranger. Ce sera en effet grâce à ses liens avec d'autres migrants et aux savoir-faire qu'il a acquis, qu'il parviendra à aider sa femme en situation migratoire.

16 Lorsqu'elles n'ont pas de conjoint déjà initié à la migration, les femmes qui partent bénéficient souvent de l'aide d'un autre membre de la famille. Il peut s'agir d'un frère, d'un cousin ou bien d'un proche du mari. Car les femmes mariées ne partent pas toujours après leur conjoint; elles peuvent être initiatrices de la migration au sein du 
couple. Cela survient lorsqu'elles ont une meilleure opportunité que leur mari d'aller travailler à l'étranger. Par exemple, une jeune femme expliquait qu'elle était partie dans la région de Milan car son beau-frère, déjà sur place, pouvait la placer comme serveuse dans un restaurant. Après en avoir discuté avec son mari, elle est partie en Italie où elle a passé neuf mois. Dans d'autres cas, la discussion porte sur "qui part » selon la disponibilité de chacun des époux. Ainsi, Anita et Bogdan, propriétaires d'un petit magasin de vêtements d'occasion, ont estimé qu'il serait plus facile pour lui de tenir la boutique et c'est donc elle qui s'est rendue en Espagne.

Lorsqu'elles ont des enfants, les femmes qui partent les laissent en général aux soins de leur grand-mère, que le père soit présent ou non en Roumanie. Mais il faut noter que cette façon de faire n'est pas particulière aux migrantes: depuis longtemps, il est fréquent que les petits-enfants soient élevés par leurs grands-parents retraités, ceci permettant aux deux parents de travailler, comme cela était en vigueur sous la dictature.

Il arrive également que des jeunes femmes célibataires se lancent dans la migration, mais cette décision est également souvent l'objet de négociation avec les parents chez qui elles habitent - ou avec d'autres membres de la famille, ce qui est moins fréquent chez les hommes. Cette différence tient notamment au financement du départ : bien que certains jeunes gens empruntent de l'argent à leurs proches, nombre cependant s'implique dans des trafics en tout genre pour rassembler les fonds nécessaires à leur départ. Il faut préciser qu'en Roumanie, l'économie informelle représente une part importante des échanges marchands ${ }^{11}$, ce qui laisse la possibilité aux personnes les plus dynamiques d'augmenter, parfois substantiellement, leurs revenus. En revanche, le départ des femmes est souvent financé par des membres de la famille, ce qui leur donne un droit de regard sur la décision et les conditions de celui-ci. Mais surtout, si la famille et les proches se préoccupent du départ d'une femme, c'est qu'ils craignent pour sa moralité, voire pour sa sécurité. La menace la plus forte pour une femme candidate au départ est d'être enrôlé dans un réseau de prostitution, lequel opère par le biais de rabatteurs ou de fausses annonces d'embauche dans les journaux (Lazaroiu et Ulrich, 2003). Dès lors, non seulement leur décision est discutée, mais leur départ dépend de la présence d'un frère, d'un cousin ou d'un proche parent qui pourra les soutenir durant leur migration. Ainsi, toutes les femmes rencontrées au cours des enquêtes bénéficiaient ou avaient bénéficié, à leurs débuts, de la protection d'un homme. Il n'organise pas toujours le voyage, mais il prodigue les conseils utiles, fournit des informations fiables et met à contribution son réseau relationnel et ses savoir-faire pour aider la migrante. En Roumanie, elle peut alors escompter l'aide d'autres migrants qui ont côtoyé son " parrain » en Occident ou bien se fier à ses amis. Parfois, le mari ou le petit ami qui est à l'étranger enverra lui-même de faux documents de voyage à sa femme ou bien lui dictera, par téléphone, comment s'y prendre pour passer une frontière ce qui lui évitera les escroqueries auxquelles sont confrontés les novices hommes et, n'ayant pas besoin d'acheter ces services au marché noir, lui permettra d'économiser sur le prix du départ. Dès lors, la migration s'organise dans des conditions moins risquées et devient plus accessible. C'est, en substance, ce qu'explique Ioana lorsqu'elle dit: "Parce que c'est très difficile si tu connais personne pour t'aider. Maintenant, comme G. est là-bas depuis longtemps, il connaît tout le monde, il sait se débrouiller. Je sais qu'il va m'aider, c'est mon frère, c'est pas pareil $»^{12}$. 
19 Ce soutien peut varier en fonction du type de lien qui lie le migrant et la nouvelle migrante mais il sera toujours important. En général, à son arrivée, la migrante est logée dans l'appartement de celui qui lui a apporté son aide. Elle y restera bien souvent, et elle pourra profiter des connaissances et de l'aide de ses amis pour la recherche d'un emploi notamment. Le soutien qu'un "protecteur " apporte à une femme dont il a la charge est d'une qualité que l'on ne retrouve que rarement entre hommes. Et même si la proximité est grande entre les hommes, notamment entre frères, on attendra d'un novice homme une autonomie plus grande et plus rapide que pour une femme.

\section{Des emplois secondaires différenciés}

20 Lors de nos enquêtes, les hommes et les femmes se retrouvaient souvent au sein de mêmes niches d'emploi : celles-ci dépendant du pôle migratoire. Ainsi, à Nice, la majorité des migrants, qu'ils soient hommes ou femmes, travaillaient en tant que vendeurs de journaux de rue. Mais lorsque la même population s'est rendue à Londres, cette opportunité n'existait pas et les migrants se sont fait embaucher dans différents secteurs, principalement dans les emplois dépréciés du tourisme. De même, quelles que soient leurs spécialités ou les places qu'ils ont pu occuper ailleurs en Europe, la très large majorité des migrants et migrantes devenaient ouvriers agricoles dans la province d'Alméria.

21 Mais si l'emploi principal d'un pôle migratoire est commun, en revanche, les emplois secondaires, qui n'occupent qu'une minorité de migrants ou migrantes, sont différenciés selon les genres. Ces emplois secondaires peuvent soit compléter ponctuellement l'activité principale soit ne concerner qu'une petite partie des migrants qui les occupent à plein-temps. Ainsi, quel que soit le lieu, les chantiers du bâtiment sont souvent une deuxième opportunité pour les hommes. Mais tandis qu'en Angleterre ils sont une source d'emploi pour de nombreux migrants, ils ne le sont que ponctuellement à Nice, et sont réservés à quelques personnes en situation régulière dans la province d'Alméria ${ }^{13}$. Les femmes investissent d'autres domaines. Elles trouvent souvent à se faire employer dans le secteur de l'entretien et des services à domicile. À Nice, un certain nombre d'entre elles complétaient les revenus de la vente des journaux en effectuant quelques heures de ménage par semaine chez des particuliers ou en apportant une assistance à des personnes âgées. Dans le sud de l'Espagne, peu travaillaient chez des particuliers, par contre une minorité avait trouvé à se faire embaucher en tant que femme de ménage dans les campings ou les hôtels de la Costa del Sol et délaissait alors les serres agricoles. À Londres, ce type d'emplois existait également, mais c'est plutôt en occupant des places de serveuses dans l'hôtellerierestauration que les emplois féminins se distinguaient de ceux des hommes.

Ces différentes occupations ne constituent pas à proprement parler des niches d'emploi dans la mesure où elles n'interviennent pas comme facteur d'attraction (Massey, 1993) dans le tracé des parcours migrants, elles sont plutôt des opportunités supplémentaires découvertes a posteriori sur un pôle migratoire. Elles sont cependant intéressantes de par leur caractère exclusivement féminin. En effet, en règle générale les migrantes obtiennent ces emplois sur la base de prospections personnelles et les occupent en dehors du milieu relationnel de leur conjoint ou du parent qui les « protège ». Il se crée alors, au sein des réseaux migrants, des sous-réseaux féminins (Degenne et Forsé, 1994), où circulent l'information sur les opportunités de travail, où les femmes se rendent 
service en se remplaçant ponctuellement ou bien travaillent ensemble. Mais il faut noter que les migrantes ne quittent pas le réseau principal pour entrer dans des univers exclusivement féminins; elles participent, en même temps, à un milieu mixte où elles sont en minorité et à des négociations et des ententes spécifiques aux femmes de ce milieu. Ces emplois secondaires apparaissent ainsi comme des ressources supplémentaires spécifiques aux portions de réseau féminines.

\section{Gagner son autonomie}

Les migrantes ont la possibilité de gagner une certaine autonomie au cours de leur migration. En effet, si leur premier départ est souvent lié au soutien d'un homme, il arrive que, par la suite, elles prennent de la distance par rapport à celui-ci et s'affirment en leur nom propre au sein du réseau dans lequel elles ont été introduites. Cette distance peut-être physique ou symbolique.

Ainsi, j'ai rencontré quelques femmes qui, tout en restant auprès de leur mari, avaient acquis une place particulière dans le cercle migrant. Elles étaient souvent citées en exemple et semblaient connues de tout le monde. Leur position centrale dans le réseau était due à une faculté particulière qu'elles mettaient au service des autres. L'une d'elles, à Nice, était une référence parmi les migrants car, ayant eu un enfant en France, elle connaissait bien les administrations et les services sociaux et pouvait donc aiguiller ses compatriotes lorsqu'ils nécessitaient de l'aide. De plus, son séjour en France a duré plusieurs années, ce qui l'a mise en contact avec plusieurs " vagues » de migrants; elle pouvait ainsi faire le lien entre les uns et les autres. De la même façon, en Espagne, une jeune femme venue rejoindre son frère avait été embauchée à l'année dans une exploitation agricole - place qui la distinguait des migrants habituellement recrutés au jour le jour - et son employeur utilisait sa médiation lorsqu'il avait besoin de personnel supplémentaire. Cette situation la mettait donc en position de fournir du travail à d'autres migrants, ce qui faisait d'elle un élément central du réseau. On constate ainsi que certaines migrantes ne vivent pas leur migration dans l'ombre des hommes; si leurs conditions de départ sont souvent identiques, après quelque temps passé en migration, elles se libèrent de la tutelle de leur protecteur soit quand un couple se défait ou lorsqu'une femme s'éloigne d'un frère ou d'un cousin. En outre la place qu'elles vont occuper par la suite dans le réseau migrant n'est pas fixe.

$\mathrm{Au}$ début, ces femmes vont profiter de la médiation de leur protecteur pour se faire une place, puis, après avoir pris des contacts, elles sont en mesure de saisir de nouvelles opportunités. Il peut s'agir de changer d'emploi, de compléter ses revenus par une nouvelle activité ou bien de partager un logement ou une voiture avec d'autres compatriotes.

Après plusieurs mois passés sur un pôle migratoire, elles ont établi des liens avec des Roumains partis sur d'autres sites et accroissent de la sorte leur potentiel migratoire. Elles s'affranchissent ainsi du soutien masculin nécessaire au départ pour agir de plus en plus de manière personnelle et indépendante et améliorer leur situation en augmentant leurs gains. Après un retour en Roumanie, elles peuvent envisager un nouveau départ, grâce au soutien d'autres personnes connues au cours de leur séjour à l'étranger. Elles enrichissent ainsi leur stock de connaissances et "se débrouillent $»^{14}$ de mieux en mieux dans l'espace informel de la migration. Elles poursuivent dès lors le même type de « carrière » que la plupart des migrants, passant de novices dépendantes 
d'autres personnes, à des pratiques diversifiées et plus autonomes. Leurs multiples relations et leur "réputation " leur permettent de collaborer avec des compatriotes, avant, parfois, de devenir expertes, ce qui n'est évidemment pas le cas de toutes les femmes, et de mettre à profit leur expérience pour elle-même et pour les autres.

\section{Une reconversion difficile lors des retours}

Pourtant, si au cours de la migration certaines femmes peuvent gagner leur autonomie et faire de la mobilité une ressource personnelle; lors des retours en Roumanie, la transformation de cette compétence en facteur d'émancipation ne se fait pas sans difficultés.

\section{Un capital symbolique fragile}

Il est tout d'abord important de comprendre la signification des retours dans ce type de migration. Après quelques mois, voire une année passée en Roumanie, nombre de ceux qui ont déjà migré envisagent à nouveau de se rendre à l'étranger. Après avoir gagné des salaires de plus de mille euros en Occident, ils ne sont plus disposés à travailler en Roumanie contre une centaine d'euros par mois ${ }^{15}$. Nombreux sont ceux qui s'installent dans la migration durant plusieurs années (Diminescu, 2000 ; Morokvasic, 1999, 2004 ; Okolski, 2001). Dès lors, leur vie se partage entre des phases de migration durant lesquelles ils accomplissent toutes sortes de tâches plus ou moins dépréciées à l'étranger et des périodes moins actives en Roumanie.

C'est durant ces retours en Roumanie que la position des femmes est le plus difficile à négocier. La migration est un faire-valoir et lors de leurs retours, beaucoup considèrent les migrants comme des aventuriers qui rentrent au pays les poches pleines. On suppose qu'ils ont réussi à s'enrichir grâce à leur débrouillardise et l'on regarde leur expérience à l'étranger comme une formation qui inspire le respect. Ils véhiculent une image de l'Occident valorisée et suscitent l'envie, souvent, de ceux qui ne sont jamais partis. Ces dernières années en Roumanie, les migrants constituent un groupe social particulièrement visible dans l'espace public. Ils jouent de leur statut et n'hésitent pas à se mettre en avant lorsque cela est possible. Il est ainsi difficile de ne pas remarquer dans les bars et les discothèques les plus occidentalisées, la présence de migrants : non seulement ce sont les plus bruyants, mais ils se distinguent aussi par l'utilisation d'interjections en français ou en anglais. On remarque également qu'ils se détachent petit à petit de leurs anciennes connaissances et se rapprochent de leurs pairs: le simple fait d'avoir appartenu à la migration crée des liens entre les migrants de retour et ils choisissent souvent pour compagnie des personnes qui, comme eux, disposent de temps libre et de suffisamment de ressources pour profiter de loisirs et ne pas travailler à temps plein. D'une part, les moyens dont ils disposent désormais leur autorisent un train de vie au-dessus de celui de la classe sociale à laquelle ils appartenaient avant leur départ. D'autre part ils affichent leur appartenance à une catégorie sociale en pleine ascension. On part pour améliorer son quotidien en Roumanie et, lorsque l'on rentre, on tient à faire savoir publiquement que cela a réussi (Potot, 2002). Mais cette attitude est surtout valorisée lorsqu'il s'agit de migrants de sexe masculin. Même si on les soupçonne parfois d'avoir des activités répréhensibles en Occident, cela n'entache que très peu leur image en Roumanie. 
30 En revanche les femmes sont toujours à la merci d'une image dépréciative liée à un manque supposé de moralité (Pheterson, 2001). Lorsqu'une femme rentre en Roumanie, elle peut gérer cette menace de différentes façons. Si elle ne veut pas faire l'objet de médisances, le plus sûr moyen est de rentrer avec son mari et de reprendre le cours de sa vie telle qu'elle l'a quittée avant son départ sans rien montrer de son ascension sociale. Si elle est restée durant tout son séjour aux côtés de son mari et qu'elle ne fréquente pas les bars ni le cercle des migrants en Roumanie, elle est peu susceptible de prêter le flanc à la critique. Par contre, lorsqu'une jeune femme se conduit comme les hommes, qu'elle se rend régulièrement - et en célibataire - dans les bars ou les discothèques, qu'elle dépense son argent de façon ostentatoire, elle provoque alors plus de médisances que d'admiration. Si elle s'est affranchie de son tuteur à l'étranger ou si elle rentre sans lui, il est alors probable que l'on soupçonne ses activités migratoires d'avoir été «immorales ». Si elle était bien intégrée à un réseau migrant, ses pairs ne saliront pas sa réputation, les attaques viendront de personnes extérieures au milieu de la migration. Par contre, si une femme s'est montrée distante par rapport aux autres migrants à l'étranger, si elle a refusé d'apporter son soutien à des compatriotes pour préserver un confort personnel ${ }^{16}$, il y a toutes les chances pour que ces derniers se montrent diffamants au sujet de ses occupations à l'Ouest. La population locale est en effet très réceptive à ces diatribes, la prostitution au départ de la Roumanie étant un phénomène bien connu ${ }^{17}$ (Pheterson, 2001). Dans un tel contexte, les jugements des migrants ont beaucoup de crédit car ils sont supposés connaître l'étranger et peuvent témoigner de ce qui s'y pratique. C'est au cours des conversations que les réputations se forgent. Il se peut que l'on affirme ouvertement, lors de soirées entre migrants, qu'une telle a fait commerce de son corps; mais il est plus fréquent de ne faire que des allusions, de dire par exemple qu'une femme a gagné beaucoup d'argent, mais qu'on ne l'a jamais vue travailler. Ou bien, si elle s'est rendue dans un pays qui ne fait pas partie des pôles migratoires domestiqués par le réseau, on suggérera que, sur ce lieu, seules les jolies jeunes femmes peuvent trouver du travail ou bien qu'elle a choisi de s'éloigner de ses pairs pour ne pas ternir sa réputation auprès des Roumains, etc.

Paradoxalement, être la cible de ce type de calomnies n'empêche pas une femme de fréquenter le cercle des migrants présents en Roumanie. En fait, le milieu reste relativement ouvert aux femmes : elles sont nombreuses à participer aux soirées dans les bars et les discothèques où se retrouvent les migrants. Pour ces femmes, toute la difficulté consistera à participer à ces manifestations publiques sans se voir rejeter par leurs proches. En effet, l'image de la femme libérée dont les migrantes ont tendance à se rapprocher reste difficile à négocier auprès de la population locale.

\section{Marginalisées dans le milieu des affaires}

Un autre aspect semble pourtant distinguer le rôle des femmes de celui des hommes; cela concerne le fonctionnement même du cercle des migrants. Il faut savoir que s'ils ne se dirigent que rarement vers un emploi salarié au retour, beaucoup d'entre eux font fructifier quelque temps leur pécule en s'engageant dans le commerce de marchandises autorisées mais dont le processus de commercialisation est illégal ou s'opère en dehors des commerces institués. Il peut s'agir de trafics liés à la migration, comme le fait d'acheter un lot de téléphones portables venus d'Occident pour le revendre au détail ou bien servir d'intermédiaire dans le cadre d'importation de vêtements d'occasion ; mais cela peut aussi concerner des opérations qui ont lieu en Roumanie, comme de racheter 
les machines d'une usine en réfection pour les revendre à bon prix. La particularité de ces trafics est qu'ils exigent d'engager des sommes supérieures à celles dont dispose la plupart des Roumains ${ }^{18}$.

L'implication dans ces multiples trafics ne suffit pas à vivre longtemps selon un train de vie faste, mais elle permet souvent aux migrants de profiter plus longtemps des gains de leur labeur à l'étranger. Il arrive aussi fréquemment que des personnes collaborent pour pouvoir s'engager dans une affaire. Dans cette façon de procéder, proche de celle, vécue en migration, le cercle migrant se trouve avantagé pour capter et tirer profit des opportunités. Mais si de nombreux migrants sont constamment engagés dans des trafics, chacun ne fait cependant part de ses projets qu'à ses partenaires ou à quelques personnes de confiance. Et tout comme à l'étranger, certaines personnes, centrales dans le groupe, se trouvent alors très sollicitées, tandis que d'autres, plus isolées, ont moins de possibilités.

Toutes ces ententes sont presque exclusivement masculines. Et même les femmes, qui parviennent à se faire accepter parmi les migrants tant à l'étranger qu'en Roumanie, n'ont quasiment aucune place dans le milieu des affaires lors des retours. D'une façon générale, les migrantes engagent rarement leurs capitaux dans ce type de transactions. Outre la difficulté de participer à ce type d'activités, les femmes contribuent plus fortement au soutien de la famille et une part importante de leur capital est ainsi absorbée (Oso, Catarino, 1997). J'ai rencontré quelques migrantes qui tentaient de faire des affaires, mais aucune ne menait à bien ce projet avec d'autres migrants. Dans ce domaine, les relations familiales jouaient un rôle prépondérant. Par exemple, une jeune femme s'engageait avec son frère dans une affaire d'importation de vêtements destinés à la revente dans l'échoppe de leur père ; une autre femme avait acheté une coupe de bois pour la revendre avec son beau-frère à des Turcs pour l'exportation. Mais ces cas ne sont pas fréquents et ne concernent pas particulièrement le milieu migrant.

Si les femmes peuvent fréquenter le cercle des migrants en Roumanie, elles n'ont guère la possibilité d'exploiter celui-ci comme une ressource dans le domaine des affaires. Leur intégration au groupe reste en cela superficielle: elles contribuent à l'image publique de celui-ci, mais sont exclues des alliances qui le fondent.

\section{Conclusion}

La position qu'occupent les femmes dans les réseaux migrants décrits est relativement originale. D'une part, on voit qu'elles s'insèrent de plus en plus dans des dispositifs migratoires mixtes. En cela, elles ne se contentent pas de rejoindre leurs hommes partis avant elles, mais participent pleinement et individuellement à la circulation migratoire. On a montré que certaines d'entre elles parviennent à utiliser un capital social transnational, acquis au cours des premiers voyages, pour acquérir une autonomie et faire usage de la mobilité pour saisir des opportunités multiples au sein d'un espace migratoire étendu. Dans ce territoire circulatoire (Tarrius, 2002), l'appartenance à la catégorie femme peut même être un atout dans la mesure où elle permet de s'inscrire dans des sous-réseaux spécifiquement féminins, porteurs de ressources.

En revanche, cette émancipation des femmes dans le processus migratoire ne trouve que partiellement écho dans la société d'origine. Si, dans le microcosme de la migration, les rapports hommes/femmes semblent s'écarter quelque peu des 
stéréotypes classiques, lors des retours, la différenciation des rôles réapparaît de manière fortement sexuée. Pour faire valoir leur ascension sociale, les femmes se trouvent confrontées à des enjeux d'image (Strauss, 1992) : si, comme cela apparaît parfois à Târgoviste, elles refusent de jouer le jeu de la discrétion, elles devront négocier une présentation d'elles-mêmes qui risque à tout moment d'être dépréciée par leur entourage, la « mauvaise réputation » étant souvent le lot de ces migrantes. Cellesci remettent en effet en question la société fortement patriarcale qu'est restée la Roumanie en proposant des modèles de comportement féminins aussi novateurs que polémiques.

\section{BIBLIOGRAPHIE}

CHECA Francisco (1999) De la Andalucia de los emigrantes a la de los inmigrantes- Diez años para la reflexión, Demofilo Revista de cultura tradicional de Andalucia, n² 29, Fundación Machado, pp. 211-255.

DEGENNE Alain, FORSÉ Michel (1994) Les réseaux sociaux. Une analyse structurale en sociologie, Paris, Armand Colin.

DIMINESCU Dana (2000) L'installation dans la mobilité : les savoir-faire migratoires des Roumains, Migrations sociétés, 13 (74), pp. 107-117.

DIMINESCU Dana (dir.) (2003) Visibles mais peu nombreux. Les circulations migratoires roumaines, Paris, Maison des Sciences de l'Hommes.

DUCHENE Gérard, ALBU Lucian Liviu, KIM Byung Yeon (2002) An attempt to estimate the size of informal economy based on household behaviour modelling, Romanian Journal of Economic Forecasting, $\mathrm{n}^{\circ}$ 1-2002, pp. 17-24.

LAGRAVE Rose-Marie, DIMINESCU Dana (1999) Faire une saison. Pour une anthropologie des migrations roumaines en France. Le cas d'Oas, Migrations Etudes, ${ }^{\circ} 91$.

LAZAROIU Sebastian, ULRICH Louis (2003) Le trafic des femmes : une perspective sociologique in, Diminescu Dana Dir., Visibles mais peu nombreux. Les circulations migratoires roumaines, pp. 265-300.

MAGYARI-VINCZE Enikö (2004) Le patriarcat d'en haut et d'en bas en Roumanie, Nouvelles Questions Féministes, Lausanne, 23 (2).

MASSEY Douglas S. (1993) Theories of International Migration : A Review and Appraisal, Population and Development Review, 19 (3), pp. 433-461.

MOROKVASIC Mirjana (1999) La mobilité transnationale comme ressource : le cas des migrants de l'Europe de l'Est, Cultures et Conflits, $\mathrm{n}^{\circ}$ 33-34, pp. 105-122.

MOROKVASIC Mirjana, EREL Umut, SHINOZAKI Kyoko Eds (2003) Crossing Borders and Shifting Boundaries. Vol. I: Gender on the move, Opladen, Leske + Budrich.

MOROKVASIC Mirjana, RUDOLPH Hedwig (dir.) (1996) Migrants. Les nouvelles mobilités en Europe, Paris, L'Harmattan. 
OKOLSKI Marek (2001) Incomplete migration. A new form of mobility in Central and Eastern Europe. The case of Polish and Ukrainian migrants, in Claire Wallace and Dariusz Stola Eds. Patterns of migration in Central Europe, Palgrave, Houndmills, pp. 105-129.

OSO Laura, CATARINO Christine (1997) Femmes chefs de ménage et migration, in Jeanne Bisilliat (dir.) Femmes du Sud et chefs de famille, Paris, Karthala, pp. 62-97.

PERALDI Michel (dir.) (2001a) Cabas et Containers. Activités marchandes informelles et réseaux migrants transfrontaliers, Paris, Maisonneuve et Larose, MMSH.

PERALDI Michel (2001b) Marseille : réseaux migrants transfrontaliers, place marchande et économie de bazar, Cultures et conflits, $\mathrm{n}^{\circ}$ 33-34.

PHETERSON Gail (1994) Droit d'asile, migration et prostitution, in Marie-Claire Caloz-Tschopp, Axel Clévenot, Maria-Pia Tschopp Asile, Violence, Exclusion en Europe, Genève, Université de Genève, pp. 57-66.

PHETERSON Gail (2001) Le prisme de la prostitution, Paris, L'Harmattan.

РОтОт Swanie (2002) Les migrants transnationaux : une nouvelle figure sociale en Roumanie, Revue d'Etudes Comparatives Est-Ouest, Paris, 33 (1), pp. 149-177.

РОтОт Swanie (2003) Quand les migrants balkaniques rencontrent ceux venus du Sud, Balkanologie, vol. VII, $\mathrm{n}^{\circ}$ 1, pp. 65-86.

SANDU Dumitru (2000) Migratia transnational a romanilor din perspective unui recensamant comunitar, Sociologie romaneasca, Bucarest, serie noua, $n^{\circ}$ 3/4-2000, pp. 5-52.

STRAUSS Anslem (1992) Miroirs et masques, Paris, Métaillé.

TARRIUS Alain (1992) Les fourmis d'Europe, Paris, L'Harmattan.

TARRIUS Alain (2002) Une forme migratoire autre : réseaux et sociétés de migrants en Méditerranée occidentale, Ville-Ecole-Intégration Enjeux, $\mathrm{n}^{\circ}$ 131, pp. 38-47.

WALDINGER Roger (1994) The making of an immigrant niche, International Migration Review, NewYork, 8 (1), Spring, pp. 3-30.

WALLACE Claire, STOLA Dariusz Eds. (2001) Patterns of migration in Central Europe, Houndmills, Palgrave.

\section{NOTES}

1. Le principe de ces migrations, qui utilisent «la mobilité comme ressource » est le même que celui des migrations polonaises étudiées par M. Morokvasic (1999).

2. Le réseau de Târgoviste a été étudié de l'hiver 1997 à l'automne 2001.

3. Jusqu'au $1^{\mathrm{er}}$ janvier 2002, les Roumains avaient l'obligation d'avoir un visa pour se déplacer dans l'Espace Schengen. Cet impératif n'existe plus aujourd'hui, mais leurs séjours doivent se limiter à trois mois et n'autorisent pas une activité salariée. Ces restrictions conduisent souvent les travailleurs migrants à entrer dans l'illégalité.

4. Au cours de l'hiver 1998, sur la centaine de vendeurs inscrits, cinquante-neuf étaient roumains et cinquante six d'entre eux étaient originaires de Târgoviste ou de sa région.

5. En tout, environ cinq mois d'investigations ont été menées auprès des migrantes et migrants dans cette ville. Lors de mon premier séjour sur place, j'étais recommandée par des personnes rencontrées à Nice et je connaissais déjà certains de mes interlocuteurs pour les avoir côtoyés en France, ce qui a facilité les contacts avec de nouvelles personnes impliquées dans la migration. 
Par la suite, j'ai effectué un séjour d'environ trois mois durant lequel j'étais logée chez la mère d'une migrante qui se trouvait alors à Nice. Cette situation m'a permis de côtoyer plusieurs de ses amies, elles-mêmes étant de retour de France ou d'autres pays. Étant l'invitée de l'une d'entre elles, j'ai pu établir des liens privilégiés avec ces femmes qui me serviront de "guides »: leur médiation m'a permis de rencontrer de nombreux migrants et migrantes et de me joindre à leur groupe lors de soirées passées dans des bars ou en discothèques.

6. La proportion de femmes migrantes était en réalité plus élevé, mais toutes ne fréquentaient pas le « groupe migrant » en Roumanie.

7. À Londres, les migrants de Târgoviste étaient plus nombreux, au moins cinq cents d'après mes informateurs, et se côtoyaient moins régulièrement. Ce pôle migratoire a fait l'objet d'une enquête de presque deux mois, à l'automne 2001, auprès des personnes originaires de Târgoviste. 8. Deux séjours de terrain d'environ deux semaines chacun en 2001 et 2002 ont permis d'enquêter dans le milieu de l'immigration dans la province espagnole.

9. Cette enquête a été menée en collaboration avec une équipe d'étudiants de l'Université de Bucarest, ce qui a permis de recueillir un grand nombre d'entretiens auprès de la population locale.

10. On peut supposer que les femmes qui entrent en migration sans s'appuyer au départ sur un capital social migrant s'orientent vers d'autres types de réseaux que ceux étudiés ici, ceux liés à la prostitution notamment, mais pas uniquement.

11. -Le secteur informel représenterait plus de la moitié des dépenses des ménages en Roumanie (Duchêne et al., 2002).

12. Discussion avec Ioana à Târgoviste, le 3-07-2000.

13. Dans cette région, le bâtiment embauche plutôt des personnes en situation régulière, laissant les clandestins travailler dans l'agriculture, alors qu'à Madrid, les Roumains sont massivement employés sur des chantiers.

14. La capacité à « se débrouiller » revenait constamment comme une compétence indispensable à la migration lors des entretiens.

15. En 2003, le salaire moyen net était, en Roumanie, de 120 euros par mois.

16. Il est fréquent, de la part des hommes comme des femmes, de s'éloigner du réseau lorsque leur situation se stabilise. Si un migrant parvient à régulariser sa situation à l'étranger par exemple, ce qu'il est en mesure d'offrir à ses compatriotes clandestins dépassera ce qu'il peut attendre d'eux, dès lors ses contacts avec le réseau se limiteront à quelques amis et il prendra de la distance avec les autres de façon à ne pas être submergé de demandes (obtention de logement, de chantiers à partager, etc.).

17. Depuis 2001, l'office des Migrations Internationales de Bucarest organise régulièrement des campagnes d'information pour prévenir le trafic humain.

18. On a souvent dit que le commerce informel était très répandu dans ce pays, mais les échanges dont il est question ici n'engagent qu'une petite partie de population, celle qui dispose des fonds nécessaires.

\section{INDEX}

Mots-clés : femmes, réseaux migratoires, réseaux sociaux, trajectoire migratoire, Roumains Index géographique : Roumanie, France, Royaume-Uni 
AUTEUR

\section{SWANIE POTOT}

Chargée de recherche CNRS, LAMES (UMR 6127), MMSH 5 rue du château de l'Horloge BP6 13094 Aix-en-Provence Cedex 2, potot@mmsh.univ-aix.fr 